\title{
Psycho-

\section{A single exposure to cocaine during development elicits regionally-selective changes in basal basic Fibroblast Growth Factor (FGF-2) gene expression and alters the trophic response to a second injection}

\begin{tabular}{|c|c|}
\hline Journal: & Psychopharmacology \\
\hline Manuscript ID: & Psych-2014-00328.R1 \\
\hline Manuscript Type: & Original Investigation \\
\hline Date Submitted by the Author: & $n / a$ \\
\hline Complete List of Authors: & $\begin{array}{l}\text { Giannotti, Giuseppe; University of Milan, Dipartimento di Scienze } \\
\text { Farmacologiche e Biomolecolari } \\
\text { Caffino, Lucia; University of Milan, Dipartimento di Scienze Farmacologiche } \\
\text { e Biomolecolari } \\
\text { Malpighi, Chiara; University of Milan, Dipartimento di Scienze } \\
\text { Farmacologiche e Biomolecolari } \\
\text { Melfi, Simona; University of Milan, Dipartimento di Scienze Farmacologiche } \\
\text { e Biomolecolari } \\
\text { Racagni, Giorgio; University of Milan, Dipartimento di Scienze } \\
\text { Farmacologiche e Biomolecolari } \\
\text { Fumagalli, Fabio; University of Milan, Dipartimento di Scienze } \\
\text { Farmacologiche e Biomolecolari }\end{array}$ \\
\hline Keywords: & ABUSE, COCAINE, NEUROTROPHIC FACTOR \\
\hline
\end{tabular}




\section{A single exposure to cocaine during development elicits regionally-selective changes in basal basic Fibroblast Growth Factor (FGF-2) gene expression and alters the trophic response to a second injection.}

Giuseppe Giannotti ${ }^{1,2 *}$, Lucia Caffino ${ }^{1,2 *}$, Chiara Malpighi ${ }^{1,2}$, Simona Melfi ${ }^{1}$, Giorgio Racagni ${ }^{1,2,3}$ and Fabio Fumagalli ${ }^{1,2}$

${ }^{1}$ Dipartimento di Scienze Farmacologiche e Biomolecolari, Università degli Studi di Milano, Via Balzaretti 9, 20133 Milano (Italy), ${ }^{2}$ Collaborative Center of Department of Antidrug Policies, Presidency of the Council of Ministers, Rome, Italy; ${ }^{3}$ I.R.C.C.S. San Giovanni di Dio-Fatebenefratelli, Brescia (Italy).

*These authors contributed equally to this manuscript and can be both considered first authors

Address correspondence to: Fabio Fumagalli, Dipartimento di Scienze Farmacologiche e Biomolecolari, Università degli Studi di Milano, Via Balzaretti 9, 20133 Milano (Italy)

Phone 39-2-50318298; Fax: 39-2-50318278; E-mail: Fabio.Fumagalli@unimi.it

\section{Acknowledgment}

This work was supported by the Dipartimento Politiche Antidroga, Presidenza del Consiglio dei Ministri, Grant CAINO 2, to Fabio Fumagalli

Financial Disclosures/Conflicts of Interest

The authors report no biomedical financial interests or potential conflicts of interest.

Short title: Cocaine at adolescence influences FGF-2 expression. 


\begin{abstract}
Rationale During adolescence, the brain is maturing and more sensitive to drugs of abuse that can influence its developmental trajectory. Recently, attention has been focused on basic Fibroblast Growth Factor (FGF-2) given that its administration early in life enhances the acquisition of cocaine self-administration and sensitization at adulthood (Turner et al., 2009; Clinton et al., 2012). Additionally, we found that abstinence from adolescent cocaine exposure long-lastingly dysregulates FGF-2 transcription (Giannotti et al., 2013).

Objectives To evaluate if 1$)$ a single injection of cocaine $(20 \mathrm{mg} / \mathrm{kg})$ at postnatal day 35 alters FGF-2 mRNA levels and 2) the first injection influences the trophic response to a second injection $(10 \mathrm{mg} / \mathrm{kg})$ provided $24 \mathrm{~h}$ or 7 days later.

Results We found regional differences in the FGF-2 expression pattern as either the first or the second injection of cocaine by themselves up-regulated FGF-2 mRNA in the medial prefrontal cortex and nucleus accumbens while down-regulating it in the hippocampus. The first injection influences the trophic response of the first. Of note, $24 \mathrm{~h}$ after the first injection, accumbal and hippocampal FGF-2 changes produced by cocaine in saline-pretreated rats were prevented in cocaine-pretreated rats. Conversely, in the medial prefrontal cortex and hippocampus 7 days after the first injection, the cocaine-induced FGF-2 changes were modified by the subsequent exposure to the psychostimulant.
\end{abstract}

Conclusions These findings show that a single cocaine injection is sufficient to produce enduring changes in the adolescent brain and indicate that early cocaine priming alters the mechanisms regulating the trophic response in a brain region-specific fashion.

Keywords: cocaine, neurotrophic factors, adolescence, FGF-2, prefrontal cortex, hippocampus 


\section{Introduction}

During adolescence, the brain is still maturing and uniquely sensitive to drugs of abuse (Burke and Miczek 2014; Stanis and Andersen 2014). Up to date, the mechanisms responsible for such higher vulnerability are still unknown. Given the role played by neurotrophic factors during critical windows of neurodevelopment, the possibility exists that perturbation of their expression or function could readily account for such vulnerability (McGinty et al. 2010; Thomas et al. 2008). The roles of neurotrophic factors in brain development range from cell proliferation, migration, differentiation and survival to synaptogenesis, myelination and neuroprotection (Reichardt 2006). However, and perhaps more important for sensitivity to drug abuse, since the functions of many of these factors overlap during development, we can not expect that changes in their expression cause overt disorders but, rather, subtle defects that may impair, at least partially, brain homeostasis and neuroplasticity.

Several lines of evidence have been accumulated in the recent years on the potential contribution of one of such trophic factors, i.e. basic fibroblast growth factor (FGF-2), to such vulnerability. FGF-2 is the prototype member of a family of heparin-binding growth factors whose contribution to brain development has been extensively documented (Riva et al. 2005). We have previously contributed to demonstrate that FGF-2 is tightly involved in the programming of neural plasticity associated with less dramatic perturbations, such as prenatal stress (Fumagalli et al. 2005) or exposure to organophosphate (Slotkin et al. 2007; 2008), suggesting that this trophic factor is sensitive to manipulations occurring early in life.

Indeed, FGF-2 might also represent a target of the early exposure to cocaine, a psychostimulant that acts primarily, but not solely, by increasing the extracellular levels of dopamine, via the blockage of the dopamine transporter (Rocha et al. 1998). In fact, FGF-2 is expressed in the developing brain (Gomez-Pinilla et al. 1994; Monfils et al. 2006) and it is modulated by dopamine (Fumagalli et al. 2003). Further, when administered early in life, it potentiated the acquisition of cocaine self-administration (Turner et al. 2009) and enhanced cocaine sensitization at adulthood (Clinton et al. 2012). In agreement with this hypothesis, we have recently shown that abstinence following adolescent exposure to cocaine leads to shortand long-term changes in FGF-2 gene expression in the rat brain (Giannotti et al. 2013). These 
data suggest that modulation of FGF-2 expression may be critical for the vulnerability of the adolescent brain to the action of cocaine.

However, the few studies on adolescent animals focused on repeated exposure to cocaine or on long-term withdrawal since the last drug administration (Caffino et al. 2013; Giannotti et al. 2014; Marin et al. 2008). In our work, we decided to evaluate 1) whether, during brain development, an acute exposure to cocaine is sufficient to change the FGF-2 transcription and 2) whether the first injection influences the trophic factor response to a second injection. Accordingly, we exposed male rats to a first injection on postnatal day (PND) 35 followed by a second injection administered 24h (PND 36) or 7 days later (PND 42). Taken together, the findings deriving from this two-hit approach will increase our knowledge on how drug abuse at adolescence might dinamically influence brain homeostasis, via changes in FGF2-mediated trophic response. 


\section{Materials and Methods}

\section{Materials}

General reagents and molecular biology reagents were purchased from Applied Biosystem (Monza, Milan, Italy), Bio-Rad Laboratories (Segrate, Milan, Italy) and Sigma (Milan, Italy). Cocaine hydrochloride was purchased from MacFarlan-Smith, Edinburgh, UK.

\section{Animals and drug treatments}

The animals used were generated by mating Sprague Dawley rats weighting $250 \mathrm{~g}$ (Charles River, Calco, Italy) and housed under standard conditions of temperature and humidity under artificial light (from 07:00 to 19:00 hours). For each set of experiments, a maximum of two male siblings was taken from each litter in order to reduce "litter effects" (Chapman and Stern 1978). Sprague Dawley male rats were exposed to the first intraperitoneal injection (i.p.) of cocaine $(20 \mathrm{mg} / \mathrm{kg}$ ) or saline at postnatal day 35 (PND 35) and then challenged with a second i.p. injection of cocaine $(10 \mathrm{mg} / \mathrm{kg})$ or saline at PND 36, i.e. 24 hours after the first one, or 7 days later at PND 42, during a period that roughly approximates adolescence in humans (Collins and Izenwasser 2004; Maldonado and Kirstein 2005). Rats were sacrificed 2 hours after the second injection.

At the scheduled time of sacrifice, brain regions of interest were immediately dissected, frozen on dry ice and stored at $-80^{\circ} \mathrm{C}$. The medial prefrontal cortex (defined as $\mathrm{Cg} 1, \mathrm{Cg} 3$, and IL subregions) and the frontal cortex corresponding to plates 5-9 of the atlas of Paxinos and Watson (1995) were dissected from 2-mm thick slices; the nucleus accumbens (including NAc-shell and NAc-core subregions) corresponding to plates 9-13 was dissected from 2-mm thick slices (Paxinos and Watson 2005). Hippocampus was grossly dissected from the whole brain.

The choice of these brain regions depended on their role in mediating the effects of cocaine: the nucleus accumbens mediates the acquiring of conditioned behaviors and plays a role in the increased sensitivity to drugs as addiction progresses; the medial prefrontal cortex modulates the emotional and motivational aspects of drug abuse; the hippocampus, instead, mediates drug-related changes in learning and memory. 
Procedures involving animals and their care were conducted in conformity with institutional guidelines that are in compliance with national (D.L. n. 116, G.U., supplement 40, 18 Febbraio, 1992, Circolare No. 8, G.U., 14 Luglio, 1994) and international laws and policies (EEC Council Directive 86/609, OJL 358, 1, December 12, 1987; Guide for the Care \& Use of Laboratory Animals, National Academies Press, 8th Edition, 2011).

\section{RNA Preparation for analysis of FGF-2 mRNA levels by Real-time RT PCR}

Total RNA was isolated and purified from the brain regions of interest as previously described (Fumagalli et al. 2013). Following total RNA extraction, the samples were processed for realtime polymerase chain reaction (PCR) to assess mRNA levels, as previously described (Fumagalli et al. 2012). Briefly, an aliquot of each sample was treated with DNase to avoid DNA contamination. RNA was analyzed by TaqMan qRT-PCR instrument (CFX384 real time system, Bio-Rad Laboratories) using the iScript ${ }^{\top \mathrm{M}}$ one-step RT-PCR kit for probes (Bio-Rad Laboratories). Samples were run in 384 well formats in triplicate as multiplexed reactions. Data were analyzed with the comparative threshold cycle $(\Delta \Delta \mathrm{Ct})$ method using $36 \mathrm{~B} 4$ as reference gene (Fumagalli et al. 2012). The following TaqMan Gene Expression Assay (Applied Biosystem) was used for FGF-2: ID Rn00570809_m1. The probe and primer sequences of for 36B4 were the following: Forward primer 5'-TTCCCACTGGCTGAAAAGGT-3', Reverse Primer 5'CGCAGCCGCAAATGC-3', Probe 5'-AAGGCCTTCCTGGCCGATCCATC-3'. Thermal cycling was initiated with an incubation at $50^{\circ} \mathrm{C}$ for $10 \mathrm{~min}$ (RNA retrotranscription) and then at $95^{\circ} \mathrm{C}$ for 5 min (Retrotranscriptase inactivation). After this initial step, 39 cycles of PCR were performed. Each PCR cycle consisted of heating the samples at $95^{\circ} \mathrm{C}$ for $10 \mathrm{~s}$ to enable the melting process and then for $30 \mathrm{~s}$ at $60^{\circ} \mathrm{C}$ for the annealing and extension reaction.

\section{Statistical analysis}

To determine treatment-related differences, we first subjected the values to a three-way ANOVA (analysis of variance), incorporating the following variables: first injection (saline vs cocaine), second injection (saline vs cocaine) and time between injections (24 hours vs 7 days). As dictated by the relevant interaction terms, low-order ANOVAs were used to determine treatment effects and interactions followed by Fischer's protected least significant 
difference (Fisher's PLSD test) to characterize differences among individual values. Significance for all tests was assumed at $p<0.05$.

\section{Results}

\section{Medial prefrontal cortex}

In the medial prefrontal cortex (mPFC), three-way ANOVA revealed an effect of the first injection $\left(F_{1,45}=8.48, p=0.006\right)$ and a first injection $\times$ second injection interaction $\left(F_{1,45}=7.03\right.$, $\mathrm{p}=0.012$ ) on FGF-2 mRNA levels (Fig. 1). No significant effect of the time between injections was instead detected $\left(F_{1,45}=2.18, p=0.149\right)$. Thus, we made intergroup comparisons by twoway ANOVAs (first injection $\times$ second injection) for each group (i.e. animals challenged with cocaine $24 \mathrm{~h}$ and 7 days after the first injection). In the 24 hour-challenged group, two-way ANOVA did not detect significant differences (first injection: $F_{1,25}=1.23, p=0.280$; second injection: $F_{1,25}=0.181, p=0.68$; first injection $x$ second injection: $F_{1,25}=2.30, p=0.145$ ). Conversely, in the 7 day-challenged group, we found effects of the first injection $\left(F_{1,20}=7.64\right.$, $p=0.014)$ and a significant first injection $x$ second injection interaction $\left(F_{1,20}=4.51, p=0.049\right)$. The post hoc analysis revealed that FGF-2 mRNA levels were increased 7 days after the first cocaine administration compared with the control group $(+57 \%, \mathrm{p}=0.003$, Fisher's PLSD test). In animals that were exposed to the first injection of cocaine, the second injection with the psychostimulant significantly decreased FGF-2 mRNA levels $(-35 \%, \mathrm{p}=0.047$, Fisher's PLSD test), an effect that was not observed in saline-pretreated animals $(+14 \%, p=0.429)$.

\section{Frontal cortex}

In the frontal cortex, three-way ANOVA of FGF-2 found only an effect of the time between injections $\left(F_{1,48}=6.89, p=0.012\right)$ (first injection: $F_{1,48}=0.556, p=0.460$; second injection: $\left.F_{1,48}=0.177, p=0.676\right)$ but the first injection of cocaine did not influence the trophic effects of the second injection (interaction between first injection $\mathrm{x}$ second injection $\mathrm{x}$ time 
between injections: $F_{1,48}=0.014, p=0.905$ ) (Fig. 2). Accordingly, we did not subdivide the statistical analysis in this region.

\section{Nucleus accumbens}

Unlike the findings for the frontal cortex, in the nucleus accumbens (NAc) three-way ANOVA revealed effects of the second injection $\left(F_{1,47}=13.59, p=0.001\right)$, first injection $x$ second injection interaction $\left(F_{1,47}=5.68, p=0.022\right)$ and first injection $x$ second injection $x$ time between injections interaction $\left(F_{1,47}=4.58, p=0.039\right)$ (Fig. 3). Examining the individual treatment effects, we found that a single cocaine injection increased FGF-2 mRNA levels 24 hours later $(+28 \%$ vs. Sal35-Sal36, $\mathrm{p}=0.010$, Fisher's PLSD test), an effect that returned to controls level 7 days after the single cocaine injection $(+5 \%$ vs. Sal35-Sal42, $p=0.682$, Fisher's PLSD test). The response to the second cocaine injection was different in saline- and cocaine-pretreated animals when challenged $24 \mathrm{~h}$ later. In fact, the cocaine-induced increase in FGF-2 mRNA levels observed in saline-pretreated rats $(+36 \%$ vs. Sal35-Sal36, $p=0.001$, Fisher's PLSD test) was prevented in cocaine-pretreated animals (-15\% vs. Coc35-Sal36, $\mathrm{p}=0.203$, Fisher's PLSD test). On the other hand, when measured 7 days later, the second injection of cocaine induced an increase independently of the pretreatment. In fact, FGF-2 mRNA levels were increased in both the saline- $(+32 \%$ vs. Sal35-Sal42, $p=0.004$, Fisher's PLSD test) and cocaine-pretreated rats ( $+29 \%$ vs. Coc35-Sal42, $\mathrm{p}=0.025$, Fisher's PLSD test).

\section{Hippocampus}

Figure $4 a$ shows the mRNA levels of FGF-2 in the hippocampus. In this brain region, three-way ANOVA revealed only a significant effect of the first injection $\mathrm{x}$ second injection interaction $\left(F_{1,45}=11.89, p=0.001\right)$ (first injection: $F_{1,45}=3.83, p=0.058$; second injection: $F_{1,45}=0.216, p=0.645 ;$ time between injections: $F_{1,45}=2.39, p=0.131$; interaction between first injection $x$ second injection $x$ time between injections: $F_{1,45}=0.657, p=0.423$ ). Examining the individual treatment effects, lower order ANOVA (first injection $\times$ second injection) were performed for each group ( $24 \mathrm{~h}$ - and 7 days-challenged). Rats that received the second injection $24 \mathrm{~h}$ later showed a significant effect of the second injection $\left(F_{1,23}=8.66, p=0.008\right)$ 
and a first injection $\times$ second injection interaction $\left(F_{1,23}=4.48, p=0.048\right)$. Twenty-four hours after the first cocaine injection, FGF-2 was significantly reduced (-22\% vs. Sal35-Sal36, $\mathrm{p}=0.038$, Fisher's PLSD test). The second injection of cocaine caused a decrease in FGF-2 mRNA levels in saline-pretreated rat (-37\% vs. Sal35-Sal36, $\mathrm{p}=0.001$, Fisher's PLSD test), but did not further reduce the FGF-2 levels in cocaine-pretreated rats (-6\% vs. Coc35-Sal36, $\mathrm{p}=0.591$, Fisher's PLSD test). Two-way ANOVA performed on the 7 day-challenged group showed an effect of the second injection $\left(F_{1,22}=8.51, p=0.009\right)$ and a first injection $\times$ second injection interaction $\left(F_{1,22}=7.30, p=0.015\right)$. FGF-2 mRNA levels were reduced 7 days after the first cocaine injection ( $-42 \%$ vs. Sal35-Sal42, $p=0.007$, Fisher's PLSD test). Cocaine challenge did not alter FGF-2 levels in saline-pretreated animals $(+2 \%$ vs. Sal35-Sal42, $p=0.873$, Fisher's LSD test), but the second cocaine injection significantly increased FGF-2 levels in cocaine-pretreated rats ( $+52 \%$ vs. Coc35-Sal42, $\mathrm{p}=0.001$, Fisher's PLSD test). 


\section{Discussion}

Our data reveal that a single exposure to cocaine during brain development is sufficient to alter FGF-2 mRNA levels in the rat brain for, at least, 7 days. Additionally, our results show that a single exposure to cocaine, during the sensitive period of adolescence, influences the response to a second dose of the psychostimulant suggesting that cocaine priming may have altered the mechanisms regulating the trophic response.

Indeed, we found regional differences in the FGF-2 response as either the first or the second injection of cocaine by themselves up-regulated FGF-2 mRNA in the MPFC and Nac while down-regulating it in the hippocampus. However, while the effect of the first injection of cocaine may be read as the acute response to cocaine, the effect brought about by the second injection, independently from the timing of the second challenge, illustrates the alteration of cell responsiveness following a cocaine-induced priming.

The analysis of the single injection of cocaine revealed a brain region-dependent profile. In the mPFC, FGF-2 mRNA levels were increased 7 days after a single injection of cocaine while no effects were observed 24 hours later, revealing a quite lasting effect promoted by the acute psychostimulant administration. In the NAc, baseline FGF-2 mRNA levels were increased 24 hours after a single injection of cocaine while returning back to control levels 7 days later. Although different in terms of temporal induction of the effect, the accumbal and cortical FGF-2 up-regulation may represent a homeostatic, protective response to oppose to cocaine-induced increase in neuronal activity and maintain cell homeostasis.

In the hippocampus, a single injection of cocaine reduced FGF-2 mRNA levels, when measured $24 \mathrm{~h}$ later, an effect that became more consistent 7 days later. This suggests that cocaine markedly reduces the hippocampal trophic response during adolescence, an effect that we did not observe at adulthood (Fumagalli et al. 2006). Such reduction might be functionally relevant. In fact, a deficit of FGF-2 expression causes defects in hippocampal neurogenesis, an effect that seems to be critical for relapse (Deschaux et al. 2012). Further, the reduction observed 7 days after the first exposure to cocaine might impair working memory, as shown by Sudai and colleagues following a similarly high dosage of cocaine (Sudai et al. 2011). 
Notably, since a single injection of cocaine is sufficient to induce anxiety in rats (Kohtz et al. 2010), an effect that has been linked to lower levels of hippocampal FGF-2 (Eren-Kocak et al. 2011; Turner et al. 2011), our data suggest that reduced FGF-2 levels in the hippocampus may represent a sign of vulnerability for cocaine-induced anxiety: this observation is intriguing since it appears that, during adolescence, acute psychostimulant exposure recapitulates anxiety-like behaviors that are usually only seen following chronic mild stress (Kompagne et al. 2008). Further experiments will explore the potential relevance of such reduction to psychiatric-like disorders.

A further degree of interest highlighted by our results is the observation that, in a brain region-dependent fashion, the first injection of cocaine has altered the trophic response to a second injection. Of note, in the Nac and hippocampus (24h) as well as in the MPFC (7d) the physiological rise of FGF-2 mRNA levels following acute exposure to cocaine is prevented in cocaine-primed animals whereas in the hippocampus (7d) we observed a marked increase of FGF-2 mRNA levels in cocaine-primed animals that was absent in animals that received the first injection of saline. Whatever the functional relevance, these results clearly indicate that cocaine priming during adolescence has profoundly dysregulated the adaptive trophic response.

We are aware that some limitations exist regarding our approach, which relies on mRNA level measurement that by itself does not represent a definitive answer about the real rate of synthesis and degradation of the protein. However, the reliability and reproducibility of the commercially available antibodies is questionable, whereas Real Time PCR is widely recognized as a reliable and reproducible technique.

In conclusion, our results show that FGF-2 is likely to be a prominent target for the developmental neurotoxicity of cocaine. The evidence that the first exposure to cocaine influences the trophic response to a second injection highlights the dynamic, perhaps maladaptive, nature of the mechanisms set into motion by the early exposure to the psychostimulant. Given the neuroprotective and neuroplastic role played by FGF-2 in the brain, we favour the possibility that cocaine-induced increase of FGF-2 mRNA levels as observed in the Nac and mPFC might be protective whereas, in the hippocampus, it may represent a factor 
2

3

4

5

6

7

8

9

10

11

12

13

14

15

16

17

18

19

20

21

22

23

24

25

26

27

28

29

30

31

32

33

34

35

36

37

38

39

40

41

42

43

44

45

46

47

48

49

50

51

52

53

54

55

56

57

58

59

60

of vulnerability, perhaps predisposing to anxiety following a week of drug withdrawal. Based on these data, we suggest that manipulations aimed at restoring FGF-2 expression could ameliorate at least some aspects of the developmental effects brought about by cocaine. 


\section{FIGURE LEGENDS}

Figure 1. Effects of a single injection of cocaine during brain development on FGF-2 mRNA levels in the rat medial prefrontal cortex: modulation by a second cocaine injection.

Adolescent male rats (PND 35) were exposed to a first injection of cocaine $(20 \mathrm{mg} / \mathrm{kg}$ ) or saline at PND 35; then, each group was divided in four different groups receiving, respectively, cocaine $(10 \mathrm{mg} / \mathrm{kg})$ or saline at PND36 or PND 42. Rats were sacrificed 2 hours after the second injection with cocaine or saline.

The results, expressed as \% of SalineP35/SalineP36 rats, represent the mean \pm S.E.M. of at least 5 independent determinations; ${ }^{* *} p<0.01$ vs. SalineP35/SalineP42 rats; ${ }^{*} p<0.05$ vs. CocaineP35/CocaineP42 rats (two-way ANOVA followed by Fisher's PLSD test). Global ANOVA analysis appears in the upper box.

Figure 2. Effects of a single injection of cocaine during brain development on FGF-2 mRNA levels in the rat frontal cortex: modulation by a second cocaine injection.

Adolescent male rats (PND 35) were exposed to a first injection of cocaine $(20 \mathrm{mg} / \mathrm{kg}$ ) or saline at PND 35; then, each group was divided in four different groups receiving, respectively, cocaine $(10 \mathrm{mg} / \mathrm{kg})$ or saline at PND 36 or PND 42. Rats were sacrificed 2 hours after the second injection with cocaine or saline.

The results, expressed as $\%$ of SalineP35/SalineP36 rats, represent the mean \pm S.E.M. of at least 5 independent determinations. Global ANOVA analysis appears in the upper box.

Figure 3. Effects of a single injection of cocaine during brain development on FGF-2 mRNA levels in the rat nucleus accumbens: modulation by a second cocaine injection.

Adolescent male rats (PND 35) were exposed to a first injection of cocaine $(20 \mathrm{mg} / \mathrm{kg}$ ) or saline at PND 35; then, each group was divided in four different groups receiving, respectively, cocaine $(10 \mathrm{mg} / \mathrm{kg})$ or saline at PND 36 or PND 42. Rats were sacrificed 2 hours after the second injection with cocaine or saline. 
The results, expressed as \% of SalineP35/SalineP36 rats, represent the mean \pm S.E.M. of at least 5 independent determinations; ${ }^{*} p<0.05,{ }^{* *} p<0.01$ vs. SalineP35/SalineP36 rats; ${ }^{\# \#} p<0.01$ vs. SalineP35/SalineP42 rats; ${ }^{\$} p<0.05$ vs. CocaineP35/SalineP42 rats (three-way ANOVA followed by Fisher's PLSD test). Global ANOVA analysis appears in the upper box.

Figure 4. Effects of a single injection of cocaine during brain development on FGF-2 mRNA levels in the rat hippocampus: modulation by a second cocaine injection.

Adolescent male rats (PND 35) were exposed to a first injection of cocaine $(20 \mathrm{mg} / \mathrm{kg}$ ) or saline at PND 35; then, each group was divided in four different groups receiving, respectively, cocaine $(10 \mathrm{mg} / \mathrm{kg})$ or saline at PND 36 or PND 42. Rats were sacrificed 2 hours after the second injection with cocaine or saline.

In panel a the results, expressed as $\%$ of SalineP35/SalineP36 rats, represent the mean \pm S.E.M. of at least 5 independent determinations; ${ }^{*} \mathrm{p}<0.05,{ }^{* *} \mathrm{p}<0.01$ vs. SalineP35/SalineP36 rats; ${ }^{\# \#} p<0.01$ vs. SalineP35/SalineP42 rats; ${ }^{\$ \$} p<0.01$ vs. CocaineP35/CocaineP42 rats (twoway ANOVA followed by Fisher's PLSD test). 


\section{References}

Burke AR, Miczek KA (2014) Stress in adolescence and drugs of abuse in rodent models: role of dopamine, CRF, and HPA axis. Psychopharmacology (Berl) 231: 1557-80

Caffino L, Calabrese F, Giannotti G, Barbon A, Verheij MM, Racagni G, Fumagalli F (2013) Stress rapidly dysregulates the glutamatergic synapse in the prefrontal cortex of cocaine-withdrawn adolescent rats. Addict Biol

Chapman RH, Stern JM (1978) Maternal stress and pituitary-adrenal manipulations during pregnancy in rats: effects on morphology and sexual behavior of male offspring. J Comp Physiol Psychol 92: 1074-83

Clinton SM, Turner CA, Flagel SB, Simpson DN, Watson SJ, Akil H (2012) Neonatal fibroblast growth factor treatment enhances cocaine sensitization. Pharmacol Biochem Behav 103: 6-17

Collins SL, Izenwasser S (2004) Chronic nicotine differentially alters cocaine-induced locomotor activity in adolescent vs. adult male and female rats. Neuropharmacology 46: 349-62

Deschaux 0, Vendruscolo LF, Schlosburg JE, Diaz-Aguilar L, Yuan CJ, Sobieraj JC, George 0, Koob GF, Mandyam CD (2012) Hippocampal neurogenesis protects against cocaineprimed relapse. Addict Biol

Eren-Kocak E, Turner CA, Watson SJ, Akil H (2011) Short-hairpin RNA silencing of endogenous fibroblast growth factor 2 in rat hippocampus increases anxiety behavior. Biol Psychiatry 69: 534-40

Fumagalli F, Bedogni F, Maragnoli ME, Gennarelli M, Perez J, Racagni G, Riva MA (2003) Dopaminergic D2 receptor activation modulates FGF-2 gene expression in rat prefrontal cortex and hippocampus. J Neurosci Res 74: 74-80

Fumagalli F, Bedogni F, Slotkin TA, Racagni G, Riva MA (2005) Prenatal stress elicits regionally-selective changes in basal FGF-2 gene expression in adulthood and alters the adult response to acute or chronic stress. Neurobiol. Dis. 20: 731-737

Fumagalli F, Calabrese F, Luoni A, Bolis F, Racagni G, Riva MA (2012) Modulation of BDNF expression by repeated treatment with the novel antipsychotic lurasidone under basal condition and in response to acute stress. Int J Neuropsychopharmacol 15: 235-46

Fumagalli F, Moro F, Caffino L, Orru A, Cassina C, Giannotti G, Di Clemente A, Racagni G, Riva MA, Cervo L (2013) Region-specific effects on BDNF expression after contingent or non-contingent cocaine i.v. self-administration in rats. Int J Neuropsychopharmacol 16: 913-8

Fumagalli F, Pasquale L, Racagni G, Riva MA (2006) Dynamic regulation of fibroblast growth factor 2 (FGF-2) gene expression in the rat brain following single and repeated cocaine administration. J Neurochem 96: 996-1004

Giannotti G, Caffino L, Calabrese F, Racagni G, Fumagalli F (2013) Dynamic modulation of basic Fibroblast Growth Factor (FGF-2) expression in the rat brain following repeated exposure to cocaine during adolescence. Psychopharmacology (Berl) 225: 553-60

Giannotti G, Caffino L, Calabrese F, Racagni G, Riva MA, Fumagalli F (2014) Prolonged abstinence from developmental cocaine exposure dysregulates BDNF and its signaling network in the medial prefrontal cortex of adult rats. Int J Neuropsychopharmacol 17: 625-34

Gomez-Pinilla F, Lee JW, Cotman CW (1994) Distribution of basic fibroblast growth factor in the developing rat brain. Neuroscience 61: 911-23 
Kohtz AS, Paris JJ, Frye CA (2010) Low doses of cocaine decrease, and high doses increase, anxiety-like behavior and brain progestogen levels among intact rats. Horm Behav 57: 474-80

Kompagne H, Bardos G, Szenasi G, Gacsalyi I, Harsing LG, Levay G (2008) Chronic mild stress generates clear depressive but ambiguous anxiety-like behaviour in rats. Behav Brain Res 193: 311-4

Maldonado AM, Kirstein CL (2005) Handling alters cocaine-induced activity in adolescent but not adult male rats. Physiol Behav 84: 321-6

Marin MT, Cruz FC, Planeta CS (2008) Cocaine-induced behavioral sensitization in adolescent rats endures until adulthood: lack of association with GluR1 and NR1 glutamate receptor subunits and tyrosine hydroxylase. Pharmacol Biochem Behav 91: 109-14

McGinty JF, Whitfield TW, Jr., Berglind WJ (2010) Brain-derived neurotrophic factor and cocaine addiction. Brain Res 1314: 183-93

Monfils MH, Driscoll I, Melvin NR, Kolb B (2006) Differential expression of basic fibroblast growth factor-2 in the developing rat brain. Neuroscience 141: 213-21

Paxinos G, Watson C (2005) The rat brain in stereotaxic coordinates, Fifth Edition. Academic Press, New York

Reichardt LF (2006) Neurotrophin-regulated signalling pathways. Phil. Trans. Royal Soc. London B 361: 1545-1564

Riva MA, Molteni R, Bedogni F, Racagni G, Fumagalli F (2005) Emerging role of the FGF system in psychiatric disorders. Trends Pharmacol Sci 26: 228-31

Rocha BA, Fumagalli F, Gainetdinov RR, Jones SR, Ator R, Giros B, Miller GW, Caron MG (1998) Cocaine self-administration in dopamine-transporter knockout mice. Nat Neurosci 1: 132-7

Slotkin TA, Seidler FJ, Fumagalli F (2007) Exposure to organophosphates reduces the expression of neurotrophic factors in neonatal rat brain regions: similarities and differences in the effects of chlorpyrifos and diazinon on the fibroblast growth factor superfamily. Environ Health Perspect 115: 909-16

Slotkin TA, Seidler FJ, Fumagalli F (2008) Targeting of neurotrophic factors, their receptors, and signaling pathways in the developmental neurotoxicity of organophosphates in vivo and in vitro. Brain Res Bull 76: 424-38

Stanis JJ, Andersen SL (2014) Reducing substance use during adolescence: a translational framework for prevention. Psychopharmacology (Berl) 231: 1437-53

Sudai E, Croitoru O, Shaldubina A, Abraham L, Gispan I, Flaumenhaft Y, Roth-Deri I, Kinor N, Aharoni S, Ben-Tzion M, Yadid G (2011) High cocaine dosage decreases neurogenesis in the hippocampus and impairs working memory. Addict Biol 16: 251-60

Thomas MJ, Kalivas PW, Shaham Y (2008) Neuroplasticity in the mesolimbic dopamine system and cocaine addiction. Br J Pharmacol 154: 327-42

Turner CA, Capriles N, Flagel SB, Perez JA, Clinton SM, Watson SJ, Akil H (2009) Neonatal FGF2 alters cocaine self-administration in the adult rat. Pharmacol Biochem Behav 92: 100-4

Turner CA, Clinton SM, Thompson RC, Watson SJ, Jr., Akil H (2011) Fibroblast growth factor-2 (FGF2) augmentation early in life alters hippocampal development and rescues the anxiety phenotype in vulnerable animals. Proc Natl Acad Sci U S A 108: 8021-5 
Fig. 1

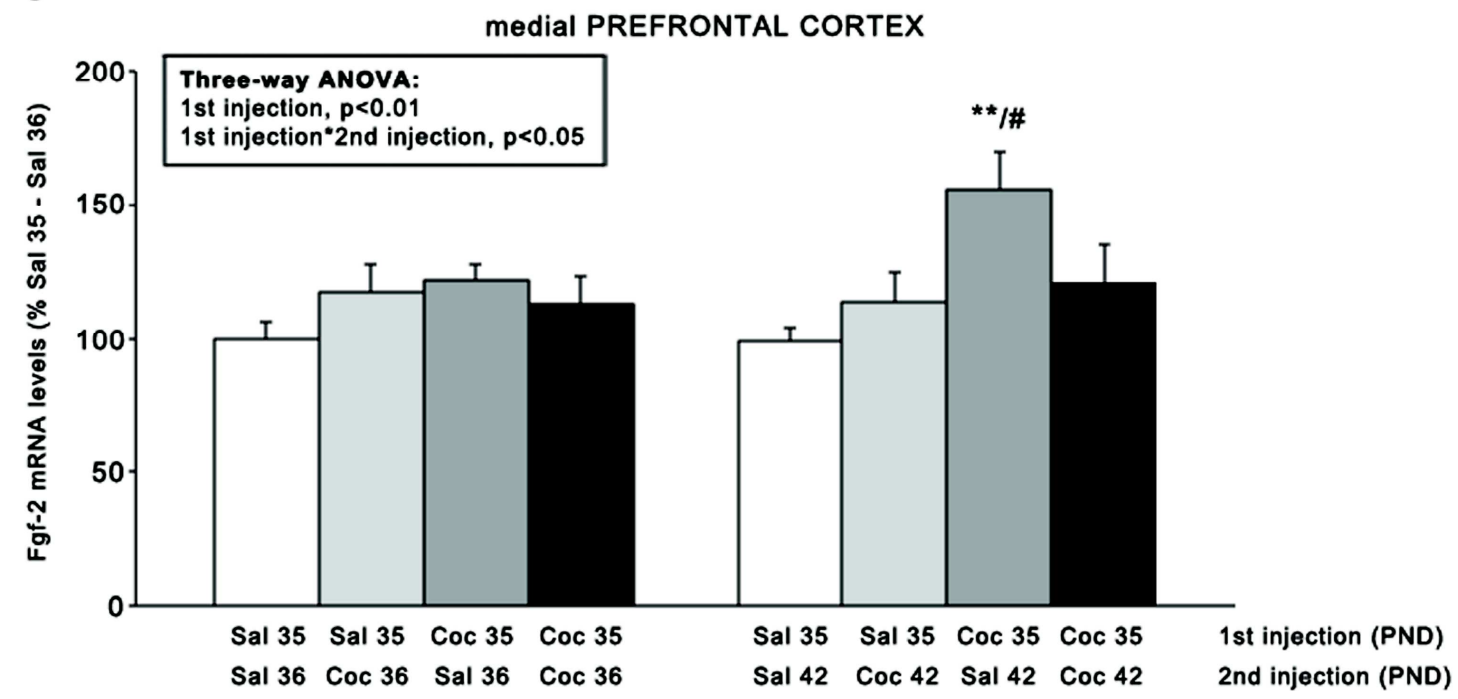

Fig. 2

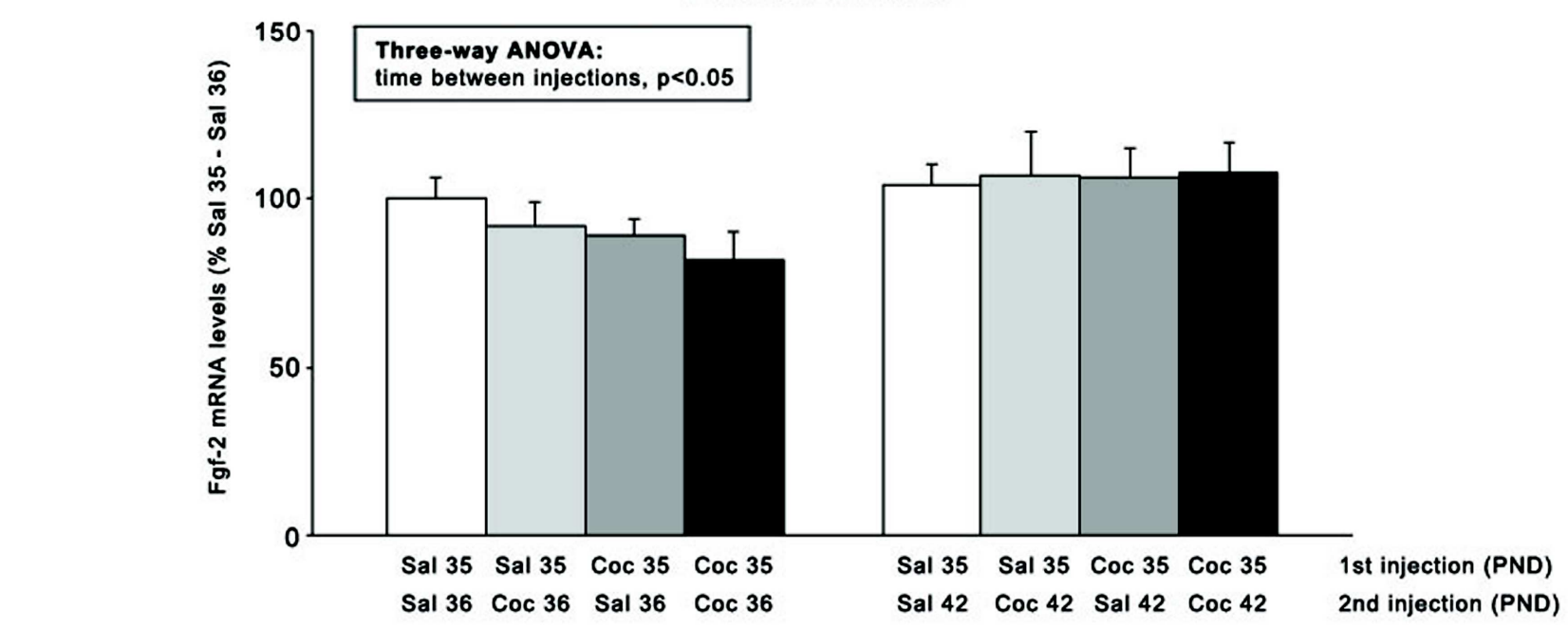

41

42

43

44

45

46

47

48

49

50

51

52

53

54

55

56

FRONTAL CORTEX

5

10


Fig. 3 NUCLEUS ACCUMBENS

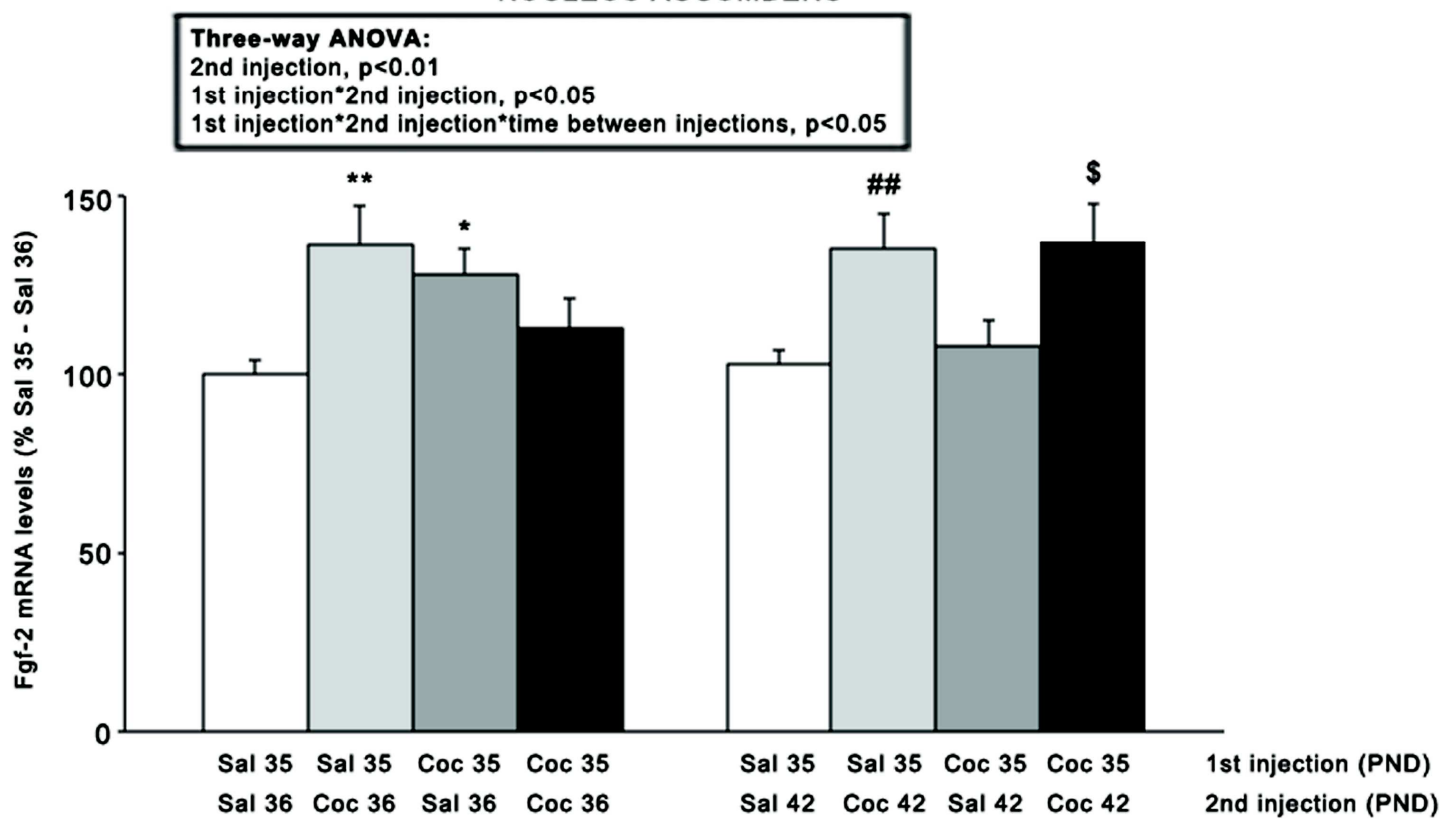

Fig. 4

HIPPOCAMPUS

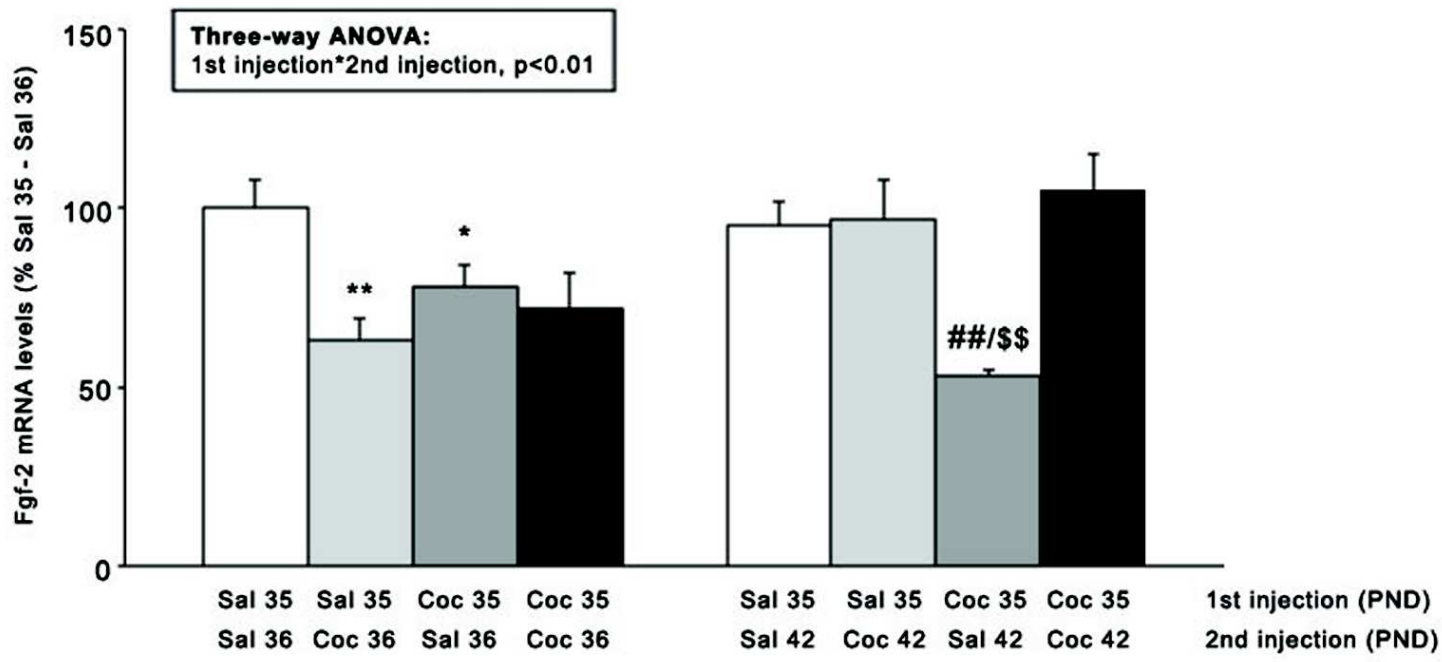

